\title{
An ab initio cluster-in-lattice model for the luminescence of $\mathrm{K}_{2} \mathrm{NbOF}_{5}$ crystal
}

\author{
Wallace D. Fragoso ${ }^{a}$, Celso de Mello Donegá ${ }^{b}$, Ricardo L. Longo ${ }^{\text {a,* }}$ \\ a Departamento de Química Fundamental, Universidade Federal de Pernambuco, Av. Prof. Luiz Freire, s/n, Cidade Universitaria, \\ 50740-540 Recife, PE, Brazil \\ ${ }^{\mathrm{b}}$ Debye Institute, Chemistry of Condensed Matter, Utrecht University, P. O. Box 80000, 3508TA Utrecht, The Netherlands
}

Received 20 January 2006; accepted 19 March 2006

\begin{abstract}
A converged model containing the $\mathrm{NbOF}_{5}^{2-}$ cluster surrounded by $10 \mathrm{~K}^{+}$ions described by effective core potentials (ECPs) embedded into 36 unit cells of (partial-)point charges was used to calculate the electronic structure of the $\mathrm{K}_{2} \mathrm{NbOF}_{5}$ crystal. These calculations were performed with several ab initio methods: CIS, TD-HF and CAS $(6,5)$ with the $6-31+\mathrm{G}(\mathrm{d})$ basis sets for O and F and the ECP-SBK basis set for $\mathrm{Nb}$ being the most adequate ones. The effects of the surrounding (partial-)point charges are significant and a proper description by ECPs of the nearest ions $\left(10 \mathrm{~K}^{+}\right)$is important, even for a qualitative model. The luminescence of the $\mathrm{K}_{2} \mathrm{NbOF}_{5}$ crystal is related to the charge-transfer transitions from the $2 \mathrm{p}$ lone-pair at the oxo ion to the $4 \mathrm{~d}^{0}$ orbitals of the $\mathrm{Nb}(\mathrm{V})$. The $4 \mathrm{~d}$ orbitals on $\mathrm{Nb}$ are more strongly perturbed as the $\mathrm{Nb}=\mathrm{O}$ (niobyl) bond distance decreases, and are shifted upwards. Whereas, changes in the $\mathrm{Nb}-\mathrm{O}$ bond distance do not affect the lowest transition energies. These results, in addition to a detailed analysis of the molecular orbitals involved in the transitions, corroborate localized behavior of the lowest transitions, which are characterized as charge-transfer bands within the niobyl moiety. A semi-quantitative description of the $\mathrm{K}_{2} \mathrm{NbOF}_{5} \cdot \mathrm{H}_{2} \mathrm{O}$ crystal photophysics was obtained with the $\mathrm{CAS}(6,5) / 6-31+\mathrm{G}(\mathrm{d})$ method, where the flatness of the ground and first excited states minima explains the uncertainties in the crystallographic position of the $\mathrm{Nb}$ ions as well as the very large broadening of the observed bands.
\end{abstract}

(C) 2006 Elsevier B.V. All rights reserved.

PACS: 71.15.Qe; 78.20.Bh; 78.55.-m; 82.20.Wt

Keywords: ECP ions; Charge-transfer transitions; Embedding; Stokes shift; CASSCF

\section{Introduction}

Luminescent materials are important due to their well known as well as potential technological applications [1]. Their efficiencies are dependent upon several radiative and non-radiative processes, which are ultimately related to their molecular or crystal structures and the electronic structure in the ground and excited states of the emitting group. Particularly, these materials usually have well

\footnotetext{
${ }^{*}$ Corresponding author. Tel.: +55 812126 8440; fax: +55 8121268442. E-mail address: longo@ufpe.br (R.L. Longo).
}

ordered crystalline structures so that the quenching of the intrinsic luminescence by extrinsic centers is avoided and thus increasing their luminescence efficiencies [1-3]. It was thus very surprising when the luminescence of niobates were observed, for the first time, in borate-lanthanum glasses [4-6]. These observations have prompted us to develop a structural model for these glasses, which could explain their luminescence. In order to accomplish such a goal in addition to a variety of experiments, some theoretical techniques should be employed. However, in order to use theoretical and computational approaches they need to be validated to provide reliable results for the electronic structure of the ground and excited states of niobates in 
crystalline or amorphous ionic environments. There are several instances where the luminescence of niobates in crystals has been assigned to the niobyl group $(\mathrm{Nb}=\mathrm{O})$ present in the (distorted) octahedral structures, that might form chains by sharing corners, edges or faces $[2,3]$. Their luminescence is highly dependent upon these superstructures, that is, if chains are formed and how they are structured. In order to develop a quantitative theoretical model we have chosen to study the isolated niobyl luminescence in the $\mathrm{K}_{2} \mathrm{NbOF}_{5}$ crystal, since its crystallographic structure has been determined by electron diffraction [7] and the photophysics of the analogue $\mathrm{K}_{2} \mathrm{NbOF}_{5} \cdot \mathrm{H}_{2} \mathrm{O}$ crystal is known [8]. It also allows the use of cluster models [9-12], since the $\mathrm{NbOF}_{5}^{2-}$ moieties are isolated by layers of $\mathrm{K}^{+}$ions [7]. We thus expect that the proper theoretical model used to describe the luminescence of $\mathrm{K}_{2} \mathrm{NbOF}_{5}$ could also be employed to study the luminescence of niobates in ionic glasses.

\section{Theoretical procedure}

The geometry of the cluster and the surrounding ionic environment has been obtained from the crystallographic data [7].

The ab initio calculations were performed with the Gaussian 98 program [13] using the standard default values for all convergences. Configuration interaction with single replacements (CIS) [14], time-dependent (TD) or polarization propagator (RPA) with Hartree-Fock (TD-HF) or hybrid functional density (TD-B3LYP) reference functions [15-19], and complete active space (CAS) [20-22] approaches have been used to calculate the electronic structure of the ground and lowest singlet and triplet excited states. The active space of the CAS method consisted of the three highest doubly occupied (HOMO, HOMO - 1, HOMO - 2) and two unoccupied (LUMO, LUMO + 2) molecular orbitals $\operatorname{CAS}(6,5)$. This active space was selected based upon the orbital symmetry, the optimization of the CAS wavefunction and practical viability of performing these calculations on larger systems. The effective core potential (ECP) SBK split valance basis [23] has been used for the $\mathrm{Nb}$ atom and the standard $6-31 \mathrm{G}, 6-31+\mathrm{G}, 6-$ $31 \mathrm{G}(\mathrm{d}), 6-31+\mathrm{G}(\mathrm{d})$ basis sets [24-28] have been employed for the $\mathrm{F}$ and $\mathrm{O}$ atoms. The ionic environment has been simulated by (partial-) point charges corresponding to the formal ionic charges of the ions in the crystal, namely, $\mathrm{Nb}=+5, \mathrm{~K}=+1, \mathrm{O}=-2$ and $\mathrm{F}=-1$, except for the ions in the boundaries which had their formal charges divided by their occupation number, the so-called Evjen-model $[29,30]$. Also, in order to avoid the unphysical distortion of the electronic density of the cluster due the point charges, the nearest $\mathrm{K}^{+}$ions were simulated by an ECP (SBK) with a core charge of 18 , no orbitals, and without the $4 s^{1}$ electron. This approach leads to fully ionic species (charge +1$)$ that have no orbitals neither electron to interfere with the transitions in the $\mathrm{NbOF}_{5}^{2-}$ cluster; but still have an ECP that can mimic the Pauli repulsion [31,32].
The calculated transition energies and oscillator strengths were fitted and normalized to a Lorentzian line shape with a given half-height band width, where care was taken when the line shape function was transformed from the frequency to the wavelength domain, since the intensities (oscillator strengths) become explicitly frequency dependent and the relative intensities can be quite different when comparing the spectrum in these domains [33].

\section{Results and discussion}

The crystallographic data [7] allowed the construction of the $\mathrm{NbOF}_{5}^{2-}$ cluster by joining two unit cells, and the most relevant interatomic distances and bond angles have been checked and matched within $0.1 \mathrm{pm}$ the available data [7]. This $\mathrm{NbOF}_{5}^{2-}$ moiety presents an isolated niobyl group $(\mathrm{Nb}=\mathrm{O})$ with a short bond distance $(168 \mathrm{pm})$ and one nonequivalent fluorine ion (axial) with a longer (208 pm) bond distance than the equatorial ions $(184 \mathrm{pm})$. However, it should be noticed that the experimental position of the $\mathrm{Nb}$ ion in the crystal has a large uncertainty of $15 \mathrm{pm}$ [7]. Fig. 1 illustrates the unit cell, including the K1 and K2 potassium ions, F1 and F2 ions, and the distorted octahedron $\mathrm{NbOF}_{5}^{2-}$ cluster.

Initially, for the $\mathrm{NbOF}_{5}^{2-}$ cluster surrounded by $10 \mathrm{~K}^{+}$ ions described by ECPs embedded into 36 unit cells of 771 (partial-)point charges (model D36) a systematic CIS study was performed in order to quantify the effects of the polarization and diffuse functions in the basis sets, which is presented in Table 1 .

It was found that the addition of the polarization functions, 6-31G(d) basis sets, shifted the lowest transitions to higher energies (shorter wavelengths, an average of $-10.5 \mathrm{~nm}$ ), whereas the inclusion of diffuse functions, $6-31+\mathrm{G}$, practically does not affect the calculated transition

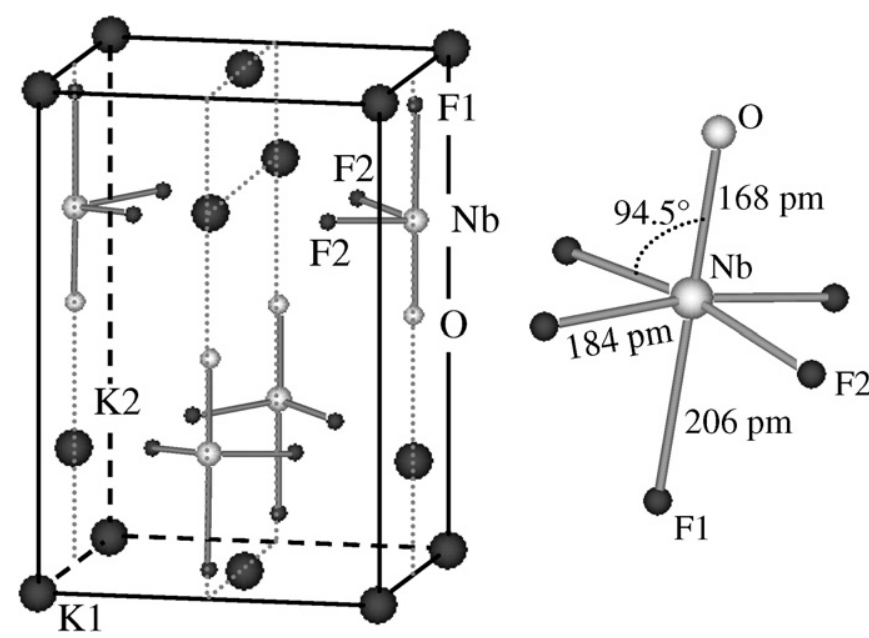

Fig. 1. The unit cell of the $\mathrm{K}_{2} \mathrm{NbOF}_{5}$ crystal (left) and the $\mathrm{NbOF}_{5}^{2-}$ cluster (right). The distinct $\mathrm{K}$ and $\mathrm{F}$ ions are indicated as $\mathrm{K} 1$ and $\mathrm{K} 2$, and as $\mathrm{F} 1$ and $\mathrm{F} 2$, respectively. In the cluster, selected bond distances and bond angle are reproduced from the crystal structure. 
Table 1

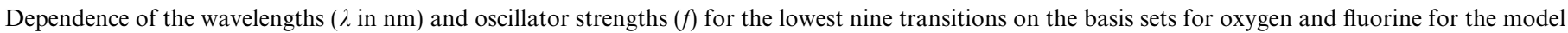
D36 calculated at the CIS level

\begin{tabular}{|c|c|c|c|c|c|c|c|c|c|c|c|c|}
\hline & \multicolumn{12}{|c|}{ Basis sets } \\
\hline & \multicolumn{3}{|c|}{$6-31 G$} & \multicolumn{3}{|c|}{$6-31+\mathrm{G}$} & \multicolumn{3}{|c|}{$6-31 G(d)$} & \multicolumn{3}{|c|}{$6-31+G(d)$} \\
\hline & $\lambda$ & $f$ & MO's & $\lambda$ & $f$ & MO's & $\lambda$ & $f$ & MO's & $\lambda$ & $f$ & MO's \\
\hline 1 & 192.6 & 0.0000 & $\begin{array}{l}33 \rightarrow 37 \\
34 \rightarrow 36\end{array}$ & 191.5 & 0.0000 & $\begin{array}{l}33 \rightarrow 37 \\
34 \rightarrow 36\end{array}$ & 179.6 & 0.0000 & $\begin{array}{l}33 \rightarrow 37 \\
34 \rightarrow 36\end{array}$ & 180.4 & 0.0000 & $\begin{array}{l}33 \rightarrow 38 \\
34 \rightarrow 37\end{array}$ \\
\hline 2 & 183.8 & 0.0000 & $\begin{array}{l}33 \rightarrow 36 \\
33 \rightarrow 37 \\
34 \rightarrow 36 \\
34 \rightarrow 37\end{array}$ & 183.1 & 0.0000 & $\begin{array}{l}33 \rightarrow 36 \\
33 \rightarrow 37 \\
34 \rightarrow 36 \\
34 \rightarrow 37\end{array}$ & 173.0 & 0.0000 & $\begin{array}{l}33 \rightarrow 36 \\
33 \rightarrow 37 \\
34 \rightarrow 36 \\
34 \rightarrow 37\end{array}$ & 173.8 & 0.0000 & $\begin{array}{l}33 \rightarrow 38 \\
34 \rightarrow 37\end{array}$ \\
\hline 3 & 183.6 & 0.0000 & $\begin{array}{l}33 \rightarrow 36 \\
33 \rightarrow 37 \\
34 \rightarrow 36 \\
34 \rightarrow 37\end{array}$ & 182.9 & 0.0000 & $\begin{array}{l}33 \rightarrow 36 \\
33 \rightarrow 37 \\
34 \rightarrow 36 \\
34 \rightarrow 37\end{array}$ & 172.8 & 0.0000 & $\begin{array}{l}33 \rightarrow 36 \\
33 \rightarrow 37 \\
34 \rightarrow 36 \\
34 \rightarrow 37\end{array}$ & 173.7 & 0.0000 & $\begin{array}{l}33 \rightarrow 37 \\
34 \rightarrow 38\end{array}$ \\
\hline 4 & 174.6 & 0.0022 & $32 \rightarrow 36$ & 173.3 & 0.0022 & $32 \rightarrow 36$ & 163.1 & 0.0018 & $\begin{array}{l}32 \rightarrow 36 \\
32 \rightarrow 40\end{array}$ & 163.3 & 0.0019 & $\begin{array}{l}32 \rightarrow 37 \\
32 \rightarrow 40 \\
32 \rightarrow 51\end{array}$ \\
\hline 5 & 174.6 & 0.0022 & $32 \rightarrow 37$ & 173.2 & 0.0022 & $32 \rightarrow 37$ & 163.1 & 0.0018 & $\begin{array}{l}32 \rightarrow 37 \\
32 \rightarrow 41\end{array}$ & 163.3 & 0.0019 & $\begin{array}{l}32 \rightarrow 38 \\
32 \rightarrow 41 \\
32 \rightarrow 52\end{array}$ \\
\hline 6 & 164.1 & 0.0011 & $34 \rightarrow 35$ & 163.6 & 0.0018 & $34 \rightarrow 35$ & 156.3 & 0.0011 & $34 \rightarrow 35$ & 157.0 & 0.0018 & $\begin{array}{l}33 \rightarrow 35 \\
34 \rightarrow 35\end{array}$ \\
\hline 7 & 164.1 & 0.0011 & $33 \rightarrow 35$ & 163.5 & 0.0018 & $33 \rightarrow 35$ & 156.3 & 0.0011 & $33 \rightarrow 35$ & 157.0 & 0.0018 & $\begin{array}{l}33 \rightarrow 35 \\
34 \rightarrow 35\end{array}$ \\
\hline 8 & 150.0 & 0.0000 & $32 \rightarrow 35$ & 149.4 & 0.0000 & $32 \rightarrow 35$ & 143.3 & 0.0000 & $32 \rightarrow 35$ & 143.7 & 0.0000 & $32 \rightarrow 35$ \\
\hline 9 & 127.7 & 0.1575 & $\begin{array}{l}32 \rightarrow 43 \\
32 \rightarrow 47 \\
33 \rightarrow 36 \\
34 \rightarrow 37\end{array}$ & 128.6 & 0.1888 & $\begin{array}{l}32 \rightarrow 45 \\
32 \rightarrow 50 \\
33 \rightarrow 36 \\
34 \rightarrow 37\end{array}$ & 124.0 & 0.1738 & $\begin{array}{l}32 \rightarrow 42 \\
32 \rightarrow 47 \\
32 \rightarrow 49 \\
33 \rightarrow 36 \\
34 \rightarrow 37\end{array}$ & 125.7 & 0.2132 & $\begin{array}{l}32 \rightarrow 44 \\
32 \rightarrow 49 \\
32 \rightarrow 53 \\
33 \rightarrow 37 \\
34 \rightarrow 38\end{array}$ \\
\hline
\end{tabular}

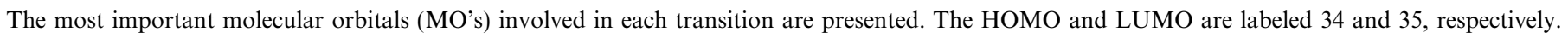

energies (an average of $-1 \mathrm{~nm}$ ), and the simultaneous inclusion of polarization and diffuse functions, $6-31+\mathrm{G}(\mathrm{d})$ basis sets, leads also to higher transition energies (an average of $-9.8 \mathrm{~nm}$ ) with respect to the $6-31 \mathrm{G}$ results. These results can be explained by the improvement in the bonding and lone-pair orbitals with the inclusion of the polarization functions. The behavior of the oscillator strengths is also quite systematic with respect to the basis sets, since the inclusion of polarization and diffuse functions usually causes an increase of ca. $30-50 \%$ in the calculated oscillator strengths for the lowest transitions. Table 1 also includes the most important molecular orbitals for the initial and final states involved in the lowest nine transitions. In order to obtain insights into the nature of these transitions the canonical HF/6-31+G(d)/SBK molecular orbitals (MO's) for the models B36 and D36 (see below) are illustrated in Figs. 2 and 3, respectively.

A comparison of Figs. 2 and 3 clearly shows the importance of the proper treatment of the nearest $\mathrm{K}^{+}$ions, mainly for the unoccupied MO's. The nature of the HOMO and HOMO - 1, namely, the 2 p lone-pairs at the oxygen atom, is retained in both models. However, the nat- ure of the LUMO changes from a diffuse orbital over the F1 atom to an almost purely localized $3 \mathrm{~d}$ orbital at the $\mathrm{Nb}$ atom when the point charges describing the nearest $\mathrm{K}^{+}$ions are replaced by ECP-ionic model. As a result, from Table 1 and Fig. 3, the lowest three transitions can be characterized as charge-transfer from the oxygen lone-pair to the $\mathrm{Nb} 3 \mathrm{~d}$ orbital, corroborating previous experimental assignments [1-3].

Despite the differences observed in Table 1 for the transition energies due to different basis sets, it should be noticed that when the calculated transition energies and oscillator strengths are fitted to Lorentzian line shape with a $10 \mathrm{~nm}$ half-height band width the spectra do not present significant differences as can be observed in Fig. 4.

Most of the features presented in the simulated spectra can be traced back to the shape and symmetry of the molecular orbitals, illustrated in Fig. 3, that have dominant contributions to the transitions. For instance, transition number 9 in Tables 1 or 2 (model D36) occurring at $125.7 \mathrm{~nm}$ has large configuration interactions involving the highest three occupied molecular orbitals and several lower unoccupied ones. On the other hand, the transition 


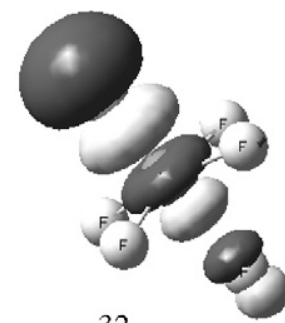

32

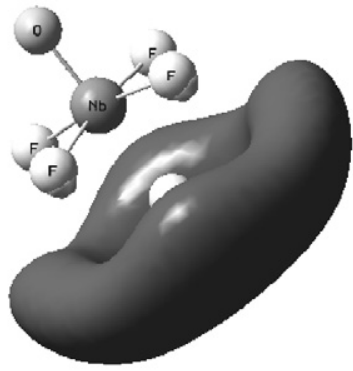

35

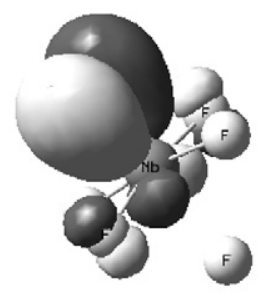

33

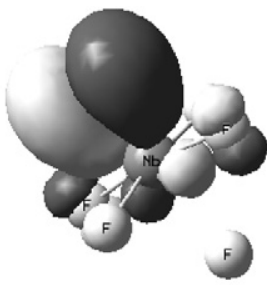

34

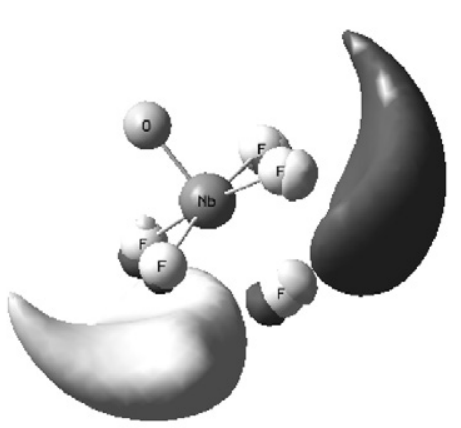

37

Fig. 2. Illustration of the canonical HF/6-31+G(d)/SBK molecular orbitals (MO's) for the B36 model: NbOF ${ }_{5}^{2-}$ cluster +36 unit cells of (partial-)point charges. The MO's are labeled such that 34 and 35 correspond to the HOMO (highest occupied MO) and LUMO (lowest unoccupied MO), respectively.
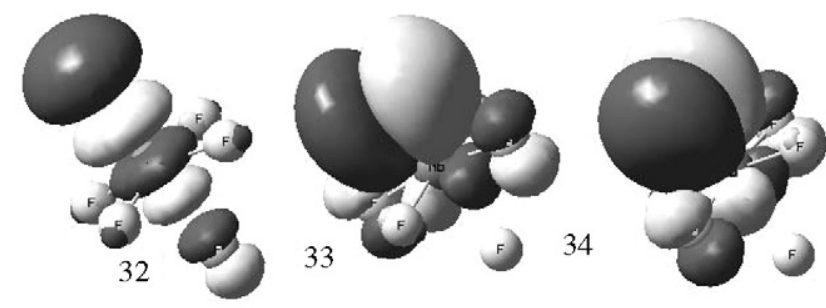

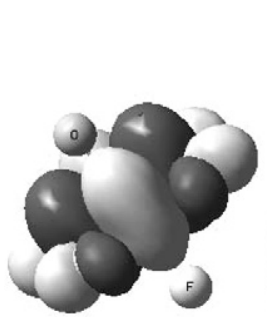

35

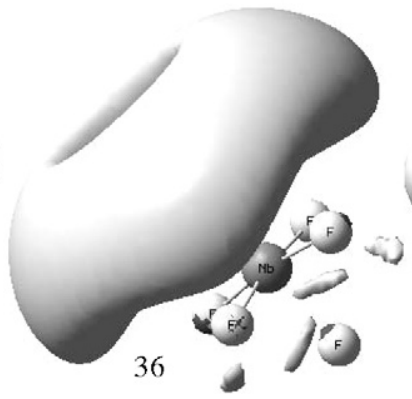

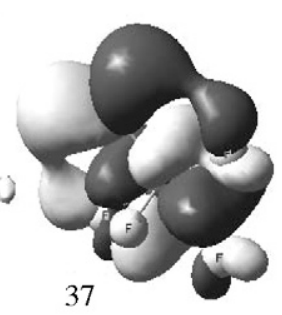

38

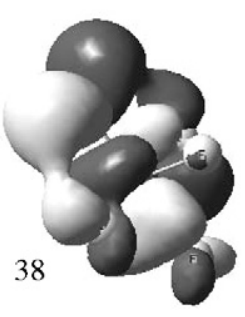

Fig. 3. Illustration of the canonical HF/6-31+G(d)/SBK molecular orbitals (MO's) for the D36 model: $\mathrm{NbOF}_{5}^{2-}$ cluster $+10 \mathrm{~K}^{+} \mathrm{ECPs}^{+} 36$ unit cells of (partial-)point charges. The MO's are labeled such that 34 and 35 correspond to the HOMO (highest occupied MO) and LUMO (lowest unoccupied MO), respectively.

number 4 , involves only the $\mathrm{HOMO}-2$ as the initial molecular orbital.

The effects of the crystal environment were simulated by (partial-)point charges surrounding the $\mathrm{NbOF}_{5}^{2-}$ cluster. This was performed by including (partial-)point charges corresponding to $2,4,6,12,18,24$ and 36 unit cells such that the final structures were as symmetric as possible, as depicted in Fig. 5.

Also, the nearest $\mathrm{K}^{+}$ions around the $\mathrm{NbOF}_{5}^{2-}$ cluster have been simulated by ECP-ionic leading to the following models: $\mathrm{NbOF}_{5}^{2-}$ isolated cluster (model A), $\mathrm{NbOF}_{5}^{2-}$ cluster plus (partial-)point charges (model $\mathrm{B} n$ ), $\mathrm{NbOF}_{5}^{2-}$ cluster plus $10 \mathrm{~K}^{+}$ECPs (model C), and $\mathrm{NbOF}_{5}^{2-}$ cluster plus 10 $\mathrm{K}^{+}$ECPs plus (partial-)point charges (model Dn), where $n=2,4,6,12,18,24$ and 36 . The CIS $/ 6-31+\mathrm{G}(\mathrm{d})$ calculated transition energies for models $\mathrm{B} n$ or $\mathrm{D} n$ showed a strong dependence for $n<12$, but remained constant (converged) for $n>24$ as can be observed in Fig. 6 .

This convergence test is important, because usually the convergence of the Madelung potential is the one tested, and there is no guarantee that its convergence will lead to converged energy transitions and intensities. It should be noticed from Figs. 5 and 6 that the results (transition energies) are practically the same for models D24 and D36, 


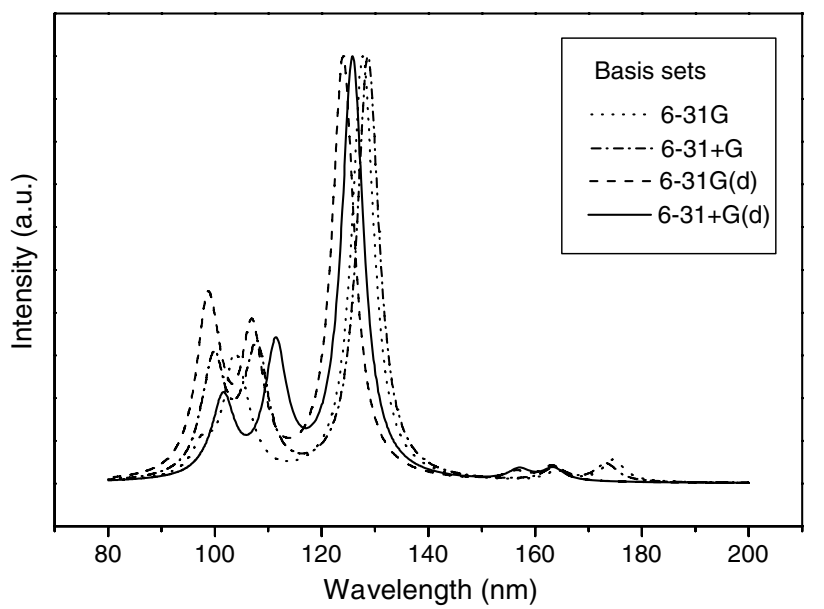

Fig. 4. Simulated absorption spectrum calculated at CIS level with different basis sets for model D36: $\mathrm{NbOF}_{5}^{2-}$ cluster $+10 \mathrm{~K}^{+} \mathrm{ECPs}+36$ unit cells of (partial-)point charges.

despite that these models do not have the same symmetry. This is due to the fact that the point charges added beyond 12 unit cells are quite far from the $\mathrm{NbOF}_{5}^{2-}$ cluster and their effects are negligible to the molecular orbital symmetry or energy. The embedding of the $\mathrm{NbOF}_{5}^{2-}$ cluster with the ionic environment has a significant effect in the transition energies since this environment affects unevenly the molecular orbital energies as well as the symmetry and localization of, mainly, the unoccupied molecular orbitals. As a result, significant differences are observed between the results obtained with the model $\mathrm{A}$ and others, as well as with the models B and D. According to Table 2, the ionic environment (ECPs and/or point charges) has the systematic effect of decreasing the transition energies, namely, the wavelengths associated with the lowest transitions increase by ca. $80 \mathrm{~nm}, 20 \mathrm{~nm}$ and $10 \mathrm{~nm}$ models B, C and $\mathrm{D}$, respectively, when compared to the transition energies in model A. It also can be observed that the oscillator strengths are drastically changed by the ionic environment. For instance, the oscillator strengths of the lower energy transitions decrease significantly for models B, C and D when compared to model A (Table 2). As a result, the ionic environment changes the absorption maxima towards shorter wavelengths, despite the low intensity transitions being present at longer wavelengths, as seen in Fig. 7. Thus, the (partial-)point charges model overestimates the crystalline effects and the ECPs for the nearest ions shield the cluster from the effects of these point charges.

However, the most significant effects of the ionic environment are upon the oscillator strengths. Despite the fact that this environment induces the appearance of excited states at lower energies, the oscillator strengths are decreased significantly, and the most intense transitions occurs at shorter wavelengths when this ionic environment is considered. More specifically, the maximum in the fitted absorption spectrum for a half-height band width of $10 \mathrm{~nm}$ is shifted from $165 \mathrm{~nm}$ for model $\mathrm{A}$, to $155 \mathrm{~nm}$ for model B36 and to $130 \mathrm{~nm}$ for models C and D36. The overall appearance of the spectra is very similar, except that the

Table 2

Dependence of the wavelengths $(\lambda)$ and oscillator strengths $(f)$ upon the models ${ }^{\mathrm{a}}$ for the $\mathrm{K}_{2} \mathrm{NbOF}_{5}$ crystal calculated at the CIS/6-31+G(d) level with SBK-ECP for $\mathrm{Nb}$

\begin{tabular}{|c|c|c|c|c|c|c|c|c|}
\hline \multirow{2}{*}{$\begin{array}{l}\text { Electronic } \\
\text { state }\end{array}$} & \multicolumn{2}{|c|}{ Model A } & \multicolumn{2}{|c|}{ Model C } & \multicolumn{2}{|c|}{ Model B36 } & \multicolumn{2}{|c|}{ Model D36 } \\
\hline & $\lambda(\mathrm{nm})$ & $f$ & $\lambda(\mathrm{nm})$ & $f$ & $\lambda(\mathrm{nm})$ & $f$ & $\lambda(\mathrm{nm})$ & $f$ \\
\hline 1 & 170.9 & 0.0214 & 192.1 & 0.0000 & 253.5 & 0.0016 & 180.4 & 0.0000 \\
\hline 4 & 156.4 & 0.0000 & 174.0 & 0.0016 & 181.8 & 0.0000 & 163.4 & 0.0019 \\
\hline 5 & 153.4 & 0.0000 & 174.0 & 0.0016 & 175.0 & 0.0000 & 163.3 & 0.0019 \\
\hline 6 & 153.2 & 0.0000 & 165.6 & 0.0017 & 174.9 & 0.0000 & 157.0 & 0.0018 \\
\hline 9 & 150.7 & 0.0058 & 128.7 & 0.1645 & 157.5 & 0.0018 & 125.7 & 0.2132 \\
\hline 10 & 149.9 & 0.0000 & 109.3 & 0.0312 & 157.5 & 0.0018 & 111.4 & 0.0414 \\
\hline 11 & 148.8 & 0.0000 & 109.3 & 0.0312 & 152.7 & 0.0000 & 111.4 & 0.0415 \\
\hline 12 & 148.5 & 0.0000 & 105.2 & 0.0047 & 150.9 & 0.0005 & 101.6 & 0.0459 \\
\hline 13 & 143.0 & 0.0008 & 105.2 & 0.0047 & 149.7 & 0.0480 & 101.6 & 0.0025 \\
\hline 14 & 143.0 & 0.0008 & 101.3 & 0.0742 & 147.5 & 0.0175 & 101.5 & 0.0021 \\
\hline 19 & 136.1 & 0.0018 & 96.8 & 0.0000 & 145.7 & 0.0000 & 97.9 & 0.0010 \\
\hline 20 & 136.1 & 0.0018 & 95.7 & 0.0396 & 143.3 & 0.0000 & 96.8 & 0.0002 \\
\hline 21 & 130.5 & 0.0308 & 95.5 & 0.0022 & 142.4 & 0.0346 & 94.9 & 0.0010 \\
\hline 22 & 130.5 & 0.0308 & 94.8 & 0.0000 & 142.4 & 0.0353 & 94.6 & 0.1175 \\
\hline
\end{tabular}

${ }^{a}$ Model A: $\mathrm{NbOF}_{5}^{2-}$ cluster; model B36: $\mathrm{NbOF}_{5}^{2-}$ cluster +36 unit cells of (partial-)point charges; model C: $\mathrm{NbOF}_{5}^{2-}$ cluster $+10 \mathrm{~K}^{+}$ECPs; model D36: $\mathrm{NbOF}_{5}^{2-}$ cluster $+10 \mathrm{~K}^{+}$ECPs +36 unit cells of (partial-)point charges. 


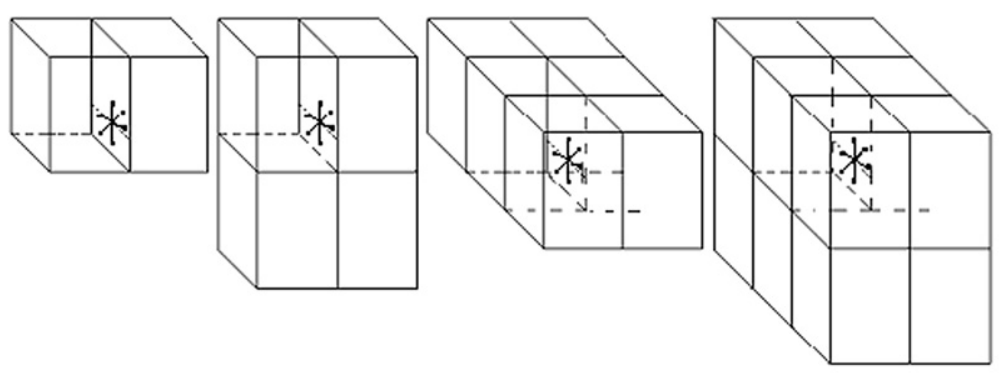

2

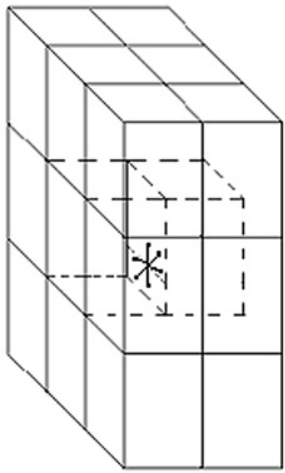

18
4

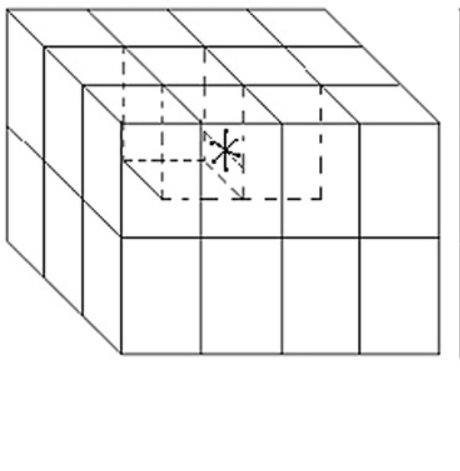

24
12

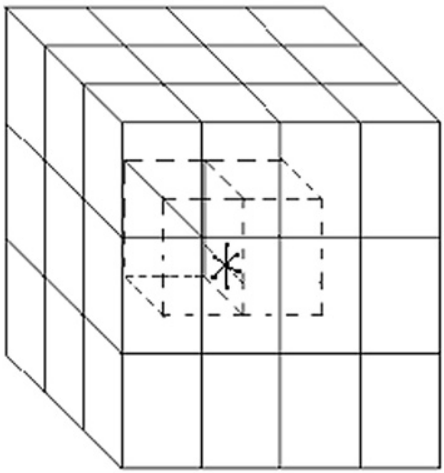

36

Fig. 5. Unit cells used in the Evjen (partial-)point charges model, with the explicit $\mathrm{NbOF}_{5}^{2-}$ cluster.

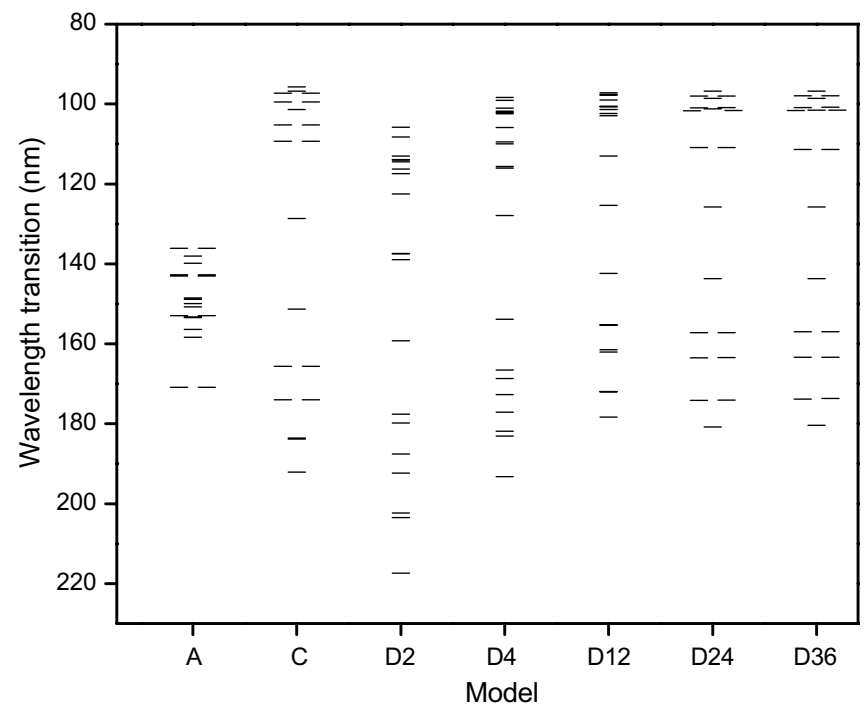

Fig. 6. The dependence of the energy levels calculated at the CIS/6$31+\mathrm{G}(\mathrm{d})$ with respect to the models for the $\mathrm{K}_{2} \mathrm{NbOF}_{5}$ crystal.

inclusion of ECPs (models $\mathrm{C}$ and $\mathrm{D} n$ ) causes broadening of the band, since many more states are packed together. From these results it can be established that it is necessary to describe the nearest ions by ECPs and that 36 unit cells, corresponding to 771 (partial-)point charges, are enough to yielded converged results for the transition energies and oscillator strengths.

It is accepted that for niobates the optical transitions are charge-transfer transitions from the $2 p$ lone-pair of the

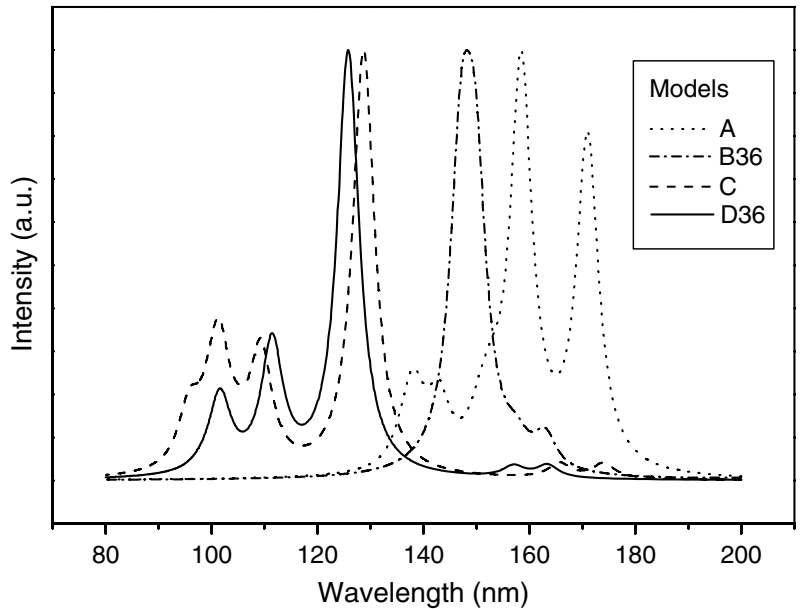

Fig. 7. Simulated absorption spectrum calculated at the CIS/6-31+G(d) with different models for the $\mathrm{K}_{2} \mathrm{NbOF}_{5}$ crystal.

oxygen ion to the $4 \mathrm{~d}^{0}$ orbitals of the $\mathrm{Nb}(\mathrm{V})$ ion [1-3], and the analysis of the lowest transitions corroborates such an assignment. Thus, it is expected that these charge-transfer transitions would be sensitive to the $\mathrm{Nb}-\mathrm{O}$ distance. To quantify this expectation, CIS/6-31+G(d) and TD-HF/6$31+\mathrm{G}(\mathrm{d})$ calculations on the model D36 were performed for $\mathrm{Nb}-\mathrm{O}$ distances equal to 165,168 and $183 \mathrm{pm}$ that are presented in Table 3. More specifically, all the ECPs and point charges as well as the oxygen and fluoride ions were kept at their crystallographic positions and only the $\mathrm{Nb}(\mathrm{V})$ ion was moved along the $\mathrm{O}=\mathrm{Nb}-\mathrm{F}_{\text {axial }}$ axis. 
Table 3

Dependence of the wavelengths $(\lambda)$ and oscillator strengths $(f)$ upon the $\mathrm{Nb}-\mathrm{O}$ distance calculated at the TD-HF/6-31+G(d) level for the model D36 of the $\mathrm{K}_{2} \mathrm{NbOF}_{5}$ crystal

\begin{tabular}{|c|c|c|c|c|c|c|}
\hline \multirow{2}{*}{$\begin{array}{l}\text { Electronic } \\
\text { state }\end{array}$} & \multicolumn{2}{|c|}{$\mathrm{Nb}-\mathrm{O}=165 \mathrm{pm}$} & \multicolumn{2}{|c|}{$\mathrm{Nb}-\mathrm{O}=168 \mathrm{pm}$} & \multicolumn{2}{|c|}{$\mathrm{Nb}-\mathrm{O}=183 \mathrm{pm}$} \\
\hline & $\lambda(\mathrm{nm})$ & $f$ & $\lambda(\mathrm{nm})$ & $f$ & $\lambda(\mathrm{nm})$ & $f$ \\
\hline 1 & 174.0 & 0.0000 & 190.4 & 0.0000 & 311.6 & 0.0000 \\
\hline 2 & 165.3 & 0.0000 & 179.4 & 0.0000 & 276.5 & 0.0000 \\
\hline 3 & 165.2 & 0.0000 & 179.4 & 0.0000 & 276.0 & 0.0000 \\
\hline 4 & 160.6 & 0.0013 & 169.5 & 0.0015 & 224.4 & 0.0018 \\
\hline 5 & 160.5 & 0.0013 & 169.4 & 0.0015 & 224.2 & 0.0018 \\
\hline 6 & 149.7 & 0.0015 & 157.4 & 0.0013 & 204.9 & 0.0008 \\
\hline 7 & 149.7 & 0.0015 & 157.4 & 0.0013 & 204.9 & 0.0008 \\
\hline 8 & 140.5 & 0.0000 & 143.9 & 0.0000 & 171.0 & 0.1823 \\
\hline 9 & 124.8 & 0.1720 & 131.6 & 0.1796 & 167.4 & 0.0000 \\
\hline 10 & 109.1 & 0.0344 & 111.5 & 0.0376 & 133.2 & 0.0119 \\
\hline 11 & 109.1 & 0.0345 & 111.5 & 0.0378 & 133.2 & 0.0120 \\
\hline 12 & 101.6 & 0.0067 & 102.8 & 0.0004 & 123.2 & 0.0187 \\
\hline 13 & 101.5 & 0.0068 & 102.8 & 0.0004 & 123.2 & 0.0186 \\
\hline 14 & 100.9 & 0.0290 & 101.8 & 0.0066 & 116.1 & 0.0151 \\
\hline
\end{tabular}

The calculations systematically yielded longer wavelengths for the lowest transitions when the $\mathrm{Nb}-\mathrm{O}$ distance increased, namely, the wavelengths increased ca. $15 \mathrm{~nm}$ when the distance increased from 165 to $168 \mathrm{pm}$, and $90 \mathrm{~nm}$ from 168 to $183 \mathrm{pm}$ for both CIS and TD-HF methods. The same kind of calculation was performed for $\mathrm{Nb}$ $\mathrm{F}_{\text {axial }}$ distance equals to 186,206 and $227 \mathrm{pm}$. The variation of the wavelengths associated with the lowest transitions was found to be smaller than $1 \mathrm{~nm}$, thus corroborating that the lowest states are highly localized at the niobyl $(\mathrm{Nb}=\mathrm{O})$ group.

It should be noted, that the values for the absorption maximum obtained with the CIS/6-31+G(d) and TD$\mathrm{HF} / 6-31+\mathrm{G}(\mathrm{d})$ methods (130 and $140 \mathrm{~nm}$, respectively) are in poor agreement with the experimental absorption maximum $(250 \mathrm{~nm})$ obtained for the $\mathrm{K}_{2} \mathrm{NbOF}_{5} \cdot \mathrm{H}_{2} \mathrm{O}$ crystal [7]. So, an approach that includes more electronic correlation effects such as the TD-B3LYP method might be adequate. However, after convergence of the self-consistent field the B3LYP method yielded several unbound electrons depending upon the model used, which resulted in unphysical transition energies. As a result, $\operatorname{CAS}(6,5) / 6-31+\mathrm{G}(\mathrm{d})$ calculations have been performed in order to obtain a better description of the lowest excited states. Unfortunately the present implementation of this method does not yield the oscillator strengths for the transitions, so only transition energies will be presented and discussed. Experimentally, the absorption maximum, the maximum of the emission $(450 \mathrm{~nm})$ and the Stokes shift $\left(18,000 \mathrm{~cm}^{-1}\right)$ have been measured for the $\mathrm{K}_{2} \mathrm{NbOF}_{5} \cdot \mathrm{H}_{2} \mathrm{O}$ crystal, so that the potential energy curves for the ground and first singlet excited states were calculated with the $\operatorname{CAS}(6,5) / 6$ $31+\mathrm{G}(\mathrm{d})$ method as a function of the $\mathrm{Nb}-\mathrm{O}$ distance for the D36 model of the $\mathrm{K}_{2} \mathrm{NbOF}_{5}$ crystal, which are presented in Fig. 8.

As can be seen both minima in the ground and excited states are very shallow and can explain the observed uncer-

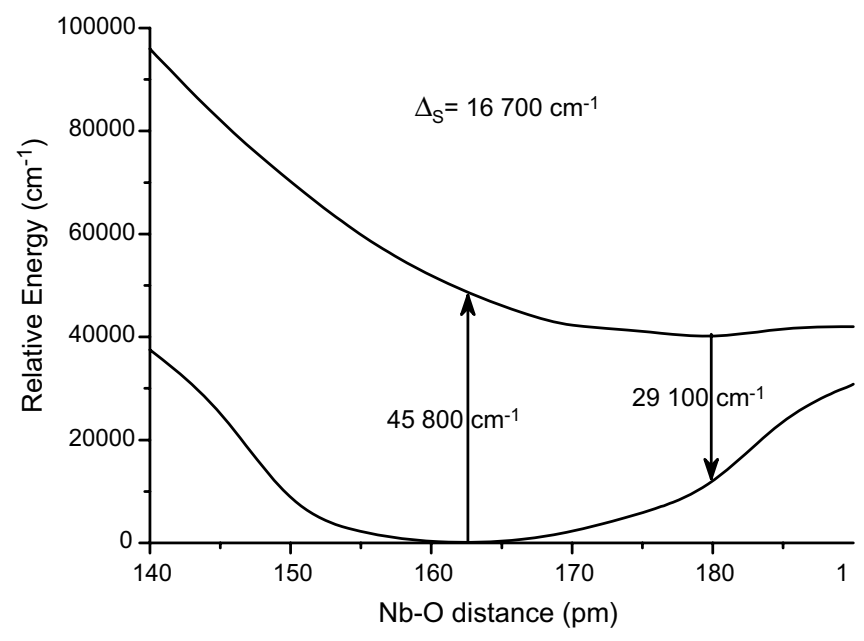

Fig. 8. Potential energy curves (ground and first excited states) for the $\mathrm{Nb}-\mathrm{O}$ distance calculated at the $\mathrm{CAS}(5,6) / 6-31+\mathrm{G}(\mathrm{d})$ level with the model D36 for the $\mathrm{K}_{2} \mathrm{NbOF}_{5}$ crystal.

tainty for the position of the $\mathrm{Nb}(\mathrm{V})$ ion in the $\mathrm{K}_{2} \mathrm{NbOF}_{5}$ crystal as well as the very broad bands observed in the absorption and luminescence spectra of the $\mathrm{K}_{2} \mathrm{NbOF}_{5} \cdot \mathrm{H}_{2} \mathrm{O}$ crystal. However, this shallowness makes the calculation of the transition energies rather difficult and somehow arbitrary, since, for instance, a variation of $\pm 5 \mathrm{pm}$ around the minimum in the ground state $(\mathrm{Nb}-\mathrm{O}=160 \mathrm{pm})$ leads to an energy variation no larger than $1800 \mathrm{~cm}^{-1}$, however, in the excited state it leads to a variation of at least $6500 \mathrm{~cm}^{-1}$. Quantitatively, it can be noted that the CAS $(6,5) / 6-31+\mathrm{G}(\mathrm{d})$ approach is far superior to the other methods for both transition energies and Stokes shift. From the CIS and TD-HF calculations it has also been noted that the oscillator strengths for the lowest transitions are very small, which might corroborate the explanation for the observed usual long decay time $(560 \mu$ s at $4.2 \mathrm{~K})$ for the luminescence of the $\mathrm{K}_{2} \mathrm{NbOF}_{5} \cdot \mathrm{H}_{2} \mathrm{O}$ crystal [3]. Also, it has been found that the calculated absorption $\left(45,800 \mathrm{~cm}^{-1}\right.$ or $236 \mathrm{~nm})$ and emission $\left(29,100 \mathrm{~cm}^{-1}\right.$ or $\left.343 \mathrm{~nm}\right)$ wavelengths are in reasonable agreement with the observed [8] transitions ( $\lambda_{\text {exc }}=250$ and $\lambda_{\mathrm{em}}=450 \mathrm{~nm}$ ) in $\mathrm{K}_{2} \mathrm{NbOF}_{5} \cdot \mathrm{H}_{2} \mathrm{O}$ crystal, as well as the Stokes shift: $16,700 \mathrm{~cm}^{-1}$ calculated and $18,000 \mathrm{~cm}^{-1}$ observed [8]. Some caution should be exercised in comparing these numbers since the experimental data were obtained for the $\mathrm{K}_{2} \mathrm{NbOF}_{5} \cdot \mathrm{H}_{2} \mathrm{O}$ crystal and the CAS approach lacks dynamical correlation, so the agreement is semi-quantitative at best. However, the qualitative conclusions presented above should hold.

\section{Conclusions}

The lowest energy states responsible for the luminescence observed in the $\mathrm{K}_{2} \mathrm{NbOF}_{5} \cdot \mathrm{H}_{2} \mathrm{O}$ crystal are quantitatively modeled by a $\mathrm{NbOF}_{5}^{2-}$ cluster embedded into a 10 $\mathrm{K}^{+}$environment described by effective core potential (ECP) for the potassium and ca. 771 (partial-)point charges with fractional charges for the boundary ions. For 
(semi)quantitative agreement it is necessary to use a CAS/ $6-31+\mathrm{G}(\mathrm{d})$ wavefunction. The calculated absorption and emission wavelengths are strongly dependent upon the crystal structure, since both ground and first excited states minima are very flat. Regarding the nature of these lowest transitions, they can be describe as charge-transfer from the oxygen ion to the $\mathrm{d}^{0} \mathrm{Nb}(\mathrm{V})$ orbitals, in agreement with the experimental assignment. As a result, these transitions have a strong dependency upon the $\mathrm{Nb}-\mathrm{O}$ distance, whereas no changes were calculated when the $\mathrm{Nb}-\mathrm{F}_{\text {axial }}$ distance was varied. These dependencies can be traced back to the destabilization of the highest occupied molecular orbital (lone-pair on the oxygen ion) as the $\mathrm{Nb}-\mathrm{O}$ distance increases.

\section{Acknowledgements}

The Brazilian agencies: CAPES, CNPq, FACEPE, FINEP, IMMC, PADCT and RENAMI are acknowledged for their partial financial support. One of the authors (W.D.F) wishes to thank CNPq and FACEPE for providing a graduate scholarship. The supercomputer center CENAPAD-SP has provided computational support.

\section{References}

[1] G. Blasse, B.C. Grabmaier, Luminescent Materials, Springer-Verlag, Berlin, 1994

[2] G. Blasse, Struct. Bonding 42 (1980) 1.

[3] G. Blasse, J. Solid State Chem. 72 (1988) 72.

[4] A.C.V. de Araújo, I.T. Weber, W.D. Fragoso, C.M. Donegá, J. Alloys Compd. 275-277 (1998) 738.

[5] W.D. Fragoso, C.M. Donegá, R.L. Longo, J. Lumin. 105 (2003) 97.

[6] W.D. Fragoso, C.M. Donegá, R.L. Longo, J. Non-Cryst. Solids 351 (2005) 3121.

[7] G.Z. Pinsker, Sov. Phys. Crystallogr. (Engl. Transl.) 11 (1967) 634.

[8] M.F. Hazenkamp, A.W.P.M. Strijbosch, G. Blasse, J. Solid State Chem. 97 (1992) 115

[9] J. Sauer, Chem. Rev. 89 (1989) 199.
[10] G.J.M. Janssen, W.C. Nieuwpoort, Phys. Rev. A 38 (1988) 3449.

[11] P.S. Bagus, F. Illas, C. Sousa, J. Chem. Phys. 100 (1994) 2943.

[12] A.L. Shluger, A.H. Harker, R.W. Grimes, C.R.A. Catlow, Phil. Trans. R. Soc. Lond. A 341 (1992) 221.

[13] M.J. Frisch, G.W. Trucks, H.B. Schlegel, G.E. Scuseria, M.A. Robb, J.R. Cheeseman, V.G. Zakrzewski, J.A. Montgomery, R.E. Stratmann, J.C. Burant, S. Dapprich, J.M. Millam, A.D. Daniels, K.N. Kudin, M.C. Strain, O. Farkas, J. Tomasi, V. Barone, M. Cossi, R. Cammi, B. Mennucci, C. Pomelli, C. Adamo, S. Clifford, J. Ochterski, G.A. Petersson, P.Y. Ayala, Q. Cui, K. Morokuma, D.K. Malick, A.D. Rabuck, K. Raghavachari, J.B. Foresman, J. Cioslowski, J.V. Ortiz, B.B. Stefanov, G. Liu, A. Liashenko, P. Piskorz, I. Komaromi, R. Gomperts, R.L. Martin, D.J. Fox, T. Keith, M.A. Al-Laham, C.Y. Peng, A. Nanayakkara, C. Gonzalez, M. Challacombe, P.M.W. Gill, B.G. Johnson, W. Chen, M.W. Wong, J.L. Andres, M. HeadGordon, E.S. Replogle, J.A. Pople, Gaussian 98 (Revision A.11), Gaussian, Inc., Pittsburgh PA, 1998.

[14] J.B. Foresman, M. Head-Gordon, J.A. Pople, M.J. Frisch, J. Phys. Chem. 96 (1992) 135.

[15] St-Amant, D.R. Salahub, Chem. Phys. Lett. 169 (1990) 387.

[16] C. Jamorski, M.E. Casida, D.R. Salahub, J. Chem. Phys. 104 (1996) 5134.

[17] R. Bauernschmitt, R. Ahlrichs, Chem. Phys. Lett. 256 (1996) 454.

[18] M.E. Casida, C. Jamorski, K.C. Casida, D.R. Salahub, J. Chem. Phys. 108 (1998) 4439.

[19] R.E. Stratmann, G.E. Scuseria, M.J. Frisch, J. Chem. Phys. 109 (1998) 8218.

[20] D. Hegarty, M.A. Robb, Mol. Phys. 38 (1979) 1795.

[21] R.H.E. Eade, M.A. Robb, Chem. Phys. Lett. 83 (1981) 362.

[22] N. Yamamoto, T. Vreven, M.A. Robb, M.J. Frisch, H.B. Schlegel, Chem. Phys. Lett. 250 (1996) 373.

[23] W. Stevens, H. Basch, J. Krauss, J. Chem. Phys. 81 (1984) 6026.

[24] R. Ditchfield, W.J. Hehre, J.A. Pople, J. Chem. Phys. 54 (1971) 724.

[25] W.J. Hehre, R. Ditchfield, J.A. Pople, J. Chem. Phys. 56 (1972) 2257.

[26] P.C. Hariharan, J.A. Pople, Mol. Phys. 27 (1974) 209.

[27] P.C. Hariharan, J.A. Pople, Theor. Chim. Acta 28 (1973) 213.

[28] M.S. Gordon, Chem. Phys. Lett. 76 (1980) 163.

[29] H.M. Evjen, Phys. Rev. 39 (1932) 675.

[30] C. Sousa, J. Casanovas, J. Rubio, F. Illas, J. Comput. Chem. 14 (1993) 680.

[31] A.Y.S. Kung, A.B. Kunz, J.M. Vail, Phys. Rev. B 26 (1982) 3352.

[32] N.W. Winter, R.M. Pitzer, D.K. Temple, J. Chem. Phys. 86 (1987) 3549.

[33] J.H. Van Vleck, V.F. Weisskopf, Rev. Mod. Phys. 17 (1945) 227. 